\title{
Laboratory Computer-Based Interventions for Better Adherence to Guidelines in the Diagnosis and Monitoring of Type 2 Diabetes
}

\author{
Maria Salinas (D) · Maite López-Garrigós · Emilio Flores • \\ Javier Lugo • Carlos Leiva-Salinas on behalf of the PRIMary Care-LABoratory (PRIMLAB) Working Group
}

Received: January 25, 2019 / Published online: March 28, 2019

(C) The Author(s) 2019

\section{ABSTRACT}

Introduction: The aim was to present two automated laboratory strategies designed to detect new cases of type 2 diabetes and prediabetes and improve their monitoring.

Methods: To improve diabetes diagnosis, we automatically registered the glycated hemoglobin (HbA1c) levels of every primary care patient between 25 and 46 years old in case of abnormal

Members of the PRIMLAB working group have given in Acknowledgements section.

Enhanced Digital Features To view enhanced digital features for this article go to https://doi.org/10.6084/ m9.figshare.7825856.

M. Salinas $(\bowtie) \cdot$ M. López-Garrigós · E. Flores · J. Lugo

Clinical Laboratory, Hospital Universitario de San Juan, San Juan de Alicante, Spain

e-mail: salinas_mar@gva.es

M. Salinas

Department of Biochemistry and Molecular Pathology, Universidad Miguel Hernandez, Elche, Spain

E. Flores

Department of Clinical Medicine, Universidad

Miguel Hernandez, Elche, Spain

C. Leiva-Salinas

Department of Radiology, University of Missouri, Columbia, MO, USA lipid testing when an HbA1c test had not been requested in the current order or during the previous year and when fasting glucose was $>100 \mathrm{mg} / \mathrm{dl}$. We counted the number of detected cases of diabetes and prediabetes and calculated the cost per identified patient. To improve diabetes monitoring, the levels of HbA1c, total cholesterol, high- and low-density lipoprotein cholesterol and triglycerides and the spot urinary albumin-to-creatinine ratios (ACRs) were automatically registered in patients with diabetes when not ordered according to guidelines. We calculated the total economic costs according to the total number of additional registered tests and reagent cost.

Results: Of 103,425 requests, 224 (0.22\%) met the inclusion criteria. Seventeen $(7.6 \%)$ patients were identified as having new cases of diabetes and $149(66.5 \%)$ of prediabetes, at a cost of $€ 15.2$ and $€ 2.3$, respectively, per case detected. From 13,874 requests in patients with diabetes, $91 \mathrm{HbA1c}$ and 708 lipid tests and 862 ACRs were automatically registered to comply with guidelines, resulting in expenses of $€ 1948.90$.

Conclusions: Making use of laboratory technology, it is possible to detect new cases of type 2 diabetes and prediabetes and to improve disease monitoring.

Keywords: Clinical guidelines adherence; Diagnosis; Laboratory strategies; Type 2 diabetes 


\section{INTRODUCTION}

The clinical laboratory plays a crucial role in the diagnosis and monitoring of type 2 diabetes. Glycated hemoglobin (HbA1c) is one of the three available tests to diagnose the disorder [1], and the marker is also used for disease monitoring. For diagnostic purposes, testing should begin at age 45 years in asymptomatic adults and should be considered in overweight or obese subjects and measured in adults who have one or more risk factors such as pathologic high-density lipoprotein cholesterol (cHDL) and/or triglyceride levels [1, 2]. For monitoring purposes, HbA1c tests should be performed twice per year in patients who meet the treatment objectives and have stable glycemic control and quarterly in patients whose treatment has changed or who do not meet the targets [1]. In addition to HbA1c tests, the management of diabetes also includes taking lipid profiles containing total cholesterol (cholesterol), low-density lipoprotein cholesterol (cLDL), cHDL and triglyceride values, as well as the urinary albumin, on an annual basis [3].

However "a global, significant, and potentially HbA1c dangerous under-request" $[4,5]$ has been reported in Spain. Additionally, REDCONLAB, a consortium to share knowledge among laboratories in Spain, also showed that urinary albumin analyses were under-requested in primary care in Spain [6]. The same pattern has also been described in other countries. Buja et al. [7] considered HbA1c, creatinine and cLDL levels measured at least once a year as indicators of diabetes care at the health system level and reported that they were adequately performed in $60.5 \%, 63.3 \%$ and $57.5 \%$ of cases, respectively, and only $44.2 \%$ of the individuals had a comprehensive assessment. In total, they showed that almost $40 \%$ of the entire diabetic population in Italy was not monitored annually for $\mathrm{HbA1c}$, creatinine or cLDL levels. Among diabetes patients in the UK, HbA1c levels had been measured in the previous year in $95.1 \%$ of the cases, cholesterol in $92.7 \%$, creatinine in 95.0\% and urinary albumin in $65.2 \%$ [8].

According to the previous evidence, there is a significant lack of compliance with diabetes guidelines $[9,10]$. Studies on general practitioner (GP) adherence rates to the guidelines suggest the usefulness of providing tools such as decision aids to accomplish this [11].

The latest technologic advances have changed the way laboratories perform. Currently, the computerized patient order entry (CPOE) is greatly improving the number of requests with patient clinical information [12]. Using this new information, diabetes mellitus was actually reported as one of the three most prevalent diagnoses prompting a laboratory test in primary care in a 7-year study [12]. In this line and by using a laboratory information system (LIS) to apply guideline recommendations, we have already proposed an automated laboratorybased strategy to detect new cases of type 2 diabetes in primary care [13].

We hypothesized that with the laboratory, and making use of the available technology, there is an opportunity to improve the detection of diabetes and prediabetes, and also diabetes monitoring, by designing and establishing interventions for better compliance with current guidelines.

The aim of the present research was to test the efficacy of two automated strategies designed in the clinical laboratory: first, to additionally identify new cases of type 2 diabetes and prediabetes through the LIS automatic register of $\mathrm{HbA} 1 \mathrm{c}$ analyses for primary care patients presenting pathologic lipid tests; second, to improve diabetes monitoring according to guideline recommendations through the LIS automatic registration of HbA1c, lipid markers and urinary albumin levels when none had been currently ordered by the GP. We finally studied the financial implications of both strategies.

\section{METHODS}

\section{Setting and Patients}

The clinical laboratory is located in a 370-bed suburban university community hospital that serves the population of the health department (HD) $(234,551$ inhabitants). 
Primary care laboratory requests are made electronically from the patient's electronic medical record (EMR) by the GPs. Laboratory requests are made through an electronic system, the CPOE, which prints the recommendations automatically in the primary care doctor's office, according to the requested tests, and offers the GPs a field to complete information on the reason for the request (clinical history/diagnostic suspicion), using the International Classification of Diseases, Ninth Revision, Clinical Modification (CIE-9-MC) codes [14]. It is not mandatory, but is optional.

The primary care patients are phlebotomized in different primary care centers (PCCs); the samples are collected by couriers and delivered to the laboratory sample reception desk. Samples arrive at the laboratory $\leq 3 \mathrm{~h}$ after phlebotomy [15].

The reports are automatically sent from the LIS to the EMR. The tests related to diabetes can be requested in an individualized manner.

We included all primary care patients of the HD covered by the clinical laboratory.

\section{Study Design}

Regarding the first objective (detecting new cases of type 2 diabetes and prediabetes through the LIS automatic HbA1c register), a prospective study was designed from 1 May 2017 to 31 May 2018.

From 1 July 2016 to 31 May 2018, a prospective study was also designed to study the second aim (improvement of diabetes monitoring through the LIS automatic HbA1c register, lipid tests and urinary albumin analyses).

\section{Laboratory Methods}

Three-milliliter blood samples were collected from each of the subjects into BD Vacutainer ${ }^{\circledR}$ $\mathrm{K}_{2}$ EDTA tubes (Becton, Dickinson and Co., Franklin Lakes, NJ, USA) to analyze HbA1c levels on a Tosoh G8 HPLC Analyzer, ion-exchange HPLC instrument [NGSP-certified, anchored to the Diabetes Control and Complication Trial (DCCT) reference study and to the IFCC Reference Method].
Glucose concentration was measured from serum collected in BD Vacutainer ${ }^{\circledR}$ serum separating tubes II Advance Tube (SST) (Becton, Dickinson and Co., Franklin Lakes, NJ, USA) using the hexokinase method with a Cobas $8000^{\circledR}$ Chemistry System (Roche Diagnostics, Basel, Switzerland).

Cholesterol, cHDL and triglyceride levels were also measured (Cobas 8000, Roche, Mannheim, Germany) in serum samples through an enzymatic colorimetric method, and cLDL was calculated using the Friedewald formula.

The quantitative urinary albumin was measured (Cobas 8000, Roche, Mannheim, Germany) with an immunoturbidimetric assay. The albumin-to-creatinine ratio (ACR) strip test is based on the protein error of the $\mathrm{pH}$ indicator (tetrabromophenol blue) and measured in UC3500 (Sysmex, Kobe, Japan).

\section{Methods}

In a first stage, a meeting was held between the laboratory professionals and the GP in charge of one of the PCCs to discuss strategies-first to find new interventions to detect new cases of type 2 diabetes and prediabetes and second to adhere to laboratory diabetes monitoring guidelines.

\section{Aim 1: Detection of New Cases of Diabetes}

Regarding the first study purpose, since a strategy for patients $>45$ years old was already established in 2013 [13], the team agreed that the LIS would automatically register HbA1c values on the laboratory request of any primary care patient $>25$ and $<46$ years old with cHDL values $<35 \mathrm{mg} / \mathrm{dl} \quad(0.90 \mathrm{mmol} / \mathrm{l})$ and/or triglyceride levels $>250 \mathrm{mg} / \mathrm{dl}(2.83 \mathrm{mmol} / \mathrm{l})$ $[1,2]$ when the former was not requested in either the current order or during the previous year and fasting glucose was $>100 \mathrm{mg} / \mathrm{dl}$. We chose the fasting glucose threshold, in consensus with GPs, because of economic constraints, since our laboratory belongs to the public sector, and because of our prior experience [13] in type 2 diabetes detection. The final diagnosis of type 2 diabetes was based on HbA1c values 
$\geq 6.5 \%(48 \mathrm{mmol} / \mathrm{mol})$ and fasting glucose $\geq 126 \mathrm{mg} / \mathrm{dl}(7 \mathrm{mmol} / \mathrm{l})$ and prediabetes when $\mathrm{HbA} 1 \mathrm{c}=5.7-6.4 \%$ and fasting glucose $=100-125 \mathrm{mg} / \mathrm{dl}$. When just one of the two tests was abnormal, the LIS would recommend a second glucose and $\mathrm{HbA} 1 \mathrm{c}$ request to confirm the presence, or not, of diabetes. In the screened population and for the detected cases, median values for HbA1c and fasting glucose were reported, to compare results between patients with diabetes and prediabetes. Finally, we counted the number of newly diagnosed cases (diabetes and prediabetes) and calculated the total economic cost per identified patient, taking into account the total number of additional HbA1c tests $(N$ HbA1c) and the cost of reagents (1.15€ per HbA1c test) (cost per identified patient $=N$ HbA1c $\times 1.15 € /$ number of identified patients).

\section{Aim 2: Improvement of Diabetes Monitoring}

To improve diabetes monitoring, the pathologists and GPs decided that HbA1c, cholesterol, CHDL, cLDC, triglyceride and ACR values would be automatically registered by the LIS in primary care patients with a laboratory order for diabetes (CIE-9-MC codes) (6) when those tests were not demanded in the current request, and also had not been previously requested in the recommended period of time, according to guideline recommendations. We reported the number of additionally registered tests according to the strategy design and their median values. We also calculated the total economic cost, taking into account the total number of additional HbA1c, lipid and ACR tests and the cost of reagents $(1.15 €$ per HbA1c test; $0.09 €$ per cholesterol test; $0.68 €$ per cHDL test, $0.24 €$ per triglyceride test and $1.31 €$ per ACR test when quantified and $0.04 €$ when just measured through strip analysis).

Thereafter, a report was sent to the GPs coordinators of the different PCCs informing them about the proposed strategies, and a 2-month reflection period was established to communicate the strategies to every GP in the nine different PCCs. In the last meeting, these strategies to detect new cases of type 2 diabetes and prediabetes and to adhere to laboratory diabetes monitoring guidelines were approved. The study was approved by the Hospital Research Committee, and the need for consent was waived.

All procedures performed in studies involving human participants were in accordance with Hospital San Juan Research Committee and with the 1964 Helsinki Declaration and its later amendments or comparable ethical standards.

\section{Statistical Analysis}

Data were statistically processed and analyzed using the SPSS 22.0 software package, and Microsoft Excel. Continuous data were tested for normal distribution using the KolmogorovSmirnov test. Descriptive statistics were presented as median and interquartile range (IQR) and percentages for continuous data and categorical data. The comparative study by two percentages was done by way of a $U$ MannWhitney test or Kruskal-Wallis test analysis, as appropriate. A two-sided $P \leq 0.05$ rule was utilized as the criterion for rejecting no difference.

\section{RESULTS}

\section{Aim 1: Detection of New Cases of Diabetes}

We obtained anonymized data for 103,425 requests for primary care patients; $224(0.22 \%)$ met the study inclusion criteria. Table 1 shows the patient characteristics. Of the 224 eligible patients, 15 (7\%) had both abnormal HbA1c $\geq 6.5 \% \quad(48 \mathrm{mmol} / \mathrm{mol})$ and fasting glucose $\geq 126 \mathrm{mg} / \mathrm{dl}(7 \mathrm{mmol} / \mathrm{l})$ and were identified as new cases of diabetes. In 17 patients, one of the two tests was abnormal and two (1\%) were diagnosed through a second request. In six cases, the second HbA1c sample did not confirm the diagnosis of diabetes. No second sample was available for the remaining nine.

The rate of diagnosis in the 224 requests among subjects between 25 and 45 years old with abnormal lipid testing was $7.6 \%$, as 17 patients were diagnosed with diabetes through the intervention. 
Table 1 Age, sex and HbAlc and fasting glucose median values, according to diabetes status

\begin{tabular}{|c|c|c|c|c|c|}
\hline & $\begin{array}{l}\text { Total } \\
(N=224)\end{array}$ & $\begin{array}{l}\text { Diabetic } \\
(N=17)\end{array}$ & $\begin{array}{l}\text { Prediabetic } \\
\text { patients } \\
(N=149)\end{array}$ & $\begin{array}{l}\text { Non-diabetic } \\
(N=58)\end{array}$ & $P$ \\
\hline Age (median, IQR) & $39(36-43)$ & $41.7(38-44)$ & $39(36-42)$ & $39(33-42)$ & 0.400 \\
\hline \multicolumn{6}{|l|}{$\operatorname{Sex}(N, \%)$} \\
\hline Male & $175(78.1 \%)$ & $13(80 \%)$ & $117(78.5 \%)$ & $45(76.7 \%)$ & \\
\hline Female & $49(21.9 \%)$ & $4(20 \%)$ & $32(21.5 \%)$ & $13(23.3 \%)$ & \\
\hline HbAlc (median, IQR) & & & & & $<0.05$ \\
\hline$\%$ & $5.71(5.46-6.01)$ & $7.86(6.98-8.41)$ & $5.83(5.57-6.01)$ & $5.46(5.31-5.58)$ & \\
\hline $\mathrm{mmol} / \mathrm{mol}$ & $39(36-42)$ & $62(53-68)$ & $40(38-42)$ & $36.2(34.5-37.5)$ & \\
\hline Glucose (median, IQR) & & & & & $<0.05$ \\
\hline $\mathrm{mg} / \mathrm{dl}$ & $\begin{array}{l}102.0 \\
(89.0-112.0)\end{array}$ & $190.5(143-272)$ & $105(101-112)$ & $83(78.0-91.0)$ & \\
\hline $\mathrm{mmol} / \mathrm{l}$ & $5.7(4.9-6.2)$ & $10.6(7.9-15.1)$ & $5.8(5.6-6.2)$ & $4.6(4.3-5.0)$ & \\
\hline $\begin{array}{l}\text { Triglicerides (median, } \\
\text { IQR) }\end{array}$ & & & & & 0.494 \\
\hline $\mathrm{mg} / \mathrm{dl}$ & $273(197-341)$ & $273(194-440)$ & $292(223-355)$ & $266(191-331)$ & \\
\hline c-HDL (median, IQR) & & & & & $<0.05$ \\
\hline $\mathrm{mg} / \mathrm{dl}$ & $32.5(29.1-35.7)$ & $28.3(25.7-33.6)$ & $31.7(29.4-35.4)$ & $33.4(29.5-39.3)$ & \\
\hline
\end{tabular}

Table 1 shows the HbA1c, fasting glucose, triglyceride and cHDL median values for patients with diabetes and prediabetes. HbA1c and glucose were significantly higher and cHDL was significantly lower than in non-diabetic patients $(P<0.05)$.

One hundred forty-nine $(66.5 \%)$ patients were diagnosed with prediabetes at a cost of $2.3 €$ per identified case.

Figure 1 shows the strategy graphically.

\section{Aim 2: Improvement of Diabetes Monitoring}

LIS captured anonymized information from 178,177 requests for primary care patients in the 2-year study period, and diabetes was the reason behind testing for 13,874 (7.8\%) requests. The median age of these patients was
68 years (P25-P75: 59-77), and 55.6\% were male.

From the 13,874 requests, 1661 (12.0\%) resulted in at least one abnormal test result. Among those automatically registered to comply with guidelines, Table 2 shows the number of tests that were automatically added through our strategy and their mean values.

With an expense of $1948.9 €$, the strategy ensured that all primary care patients who underwent laboratory testing because of diabetes met clinical guidelines.

\section{DISCUSSION}

Automated laboratory strategies, through the additional tests requested and performed and results reported, can detect new cases of diabetes and prediabetes in a certain patient 


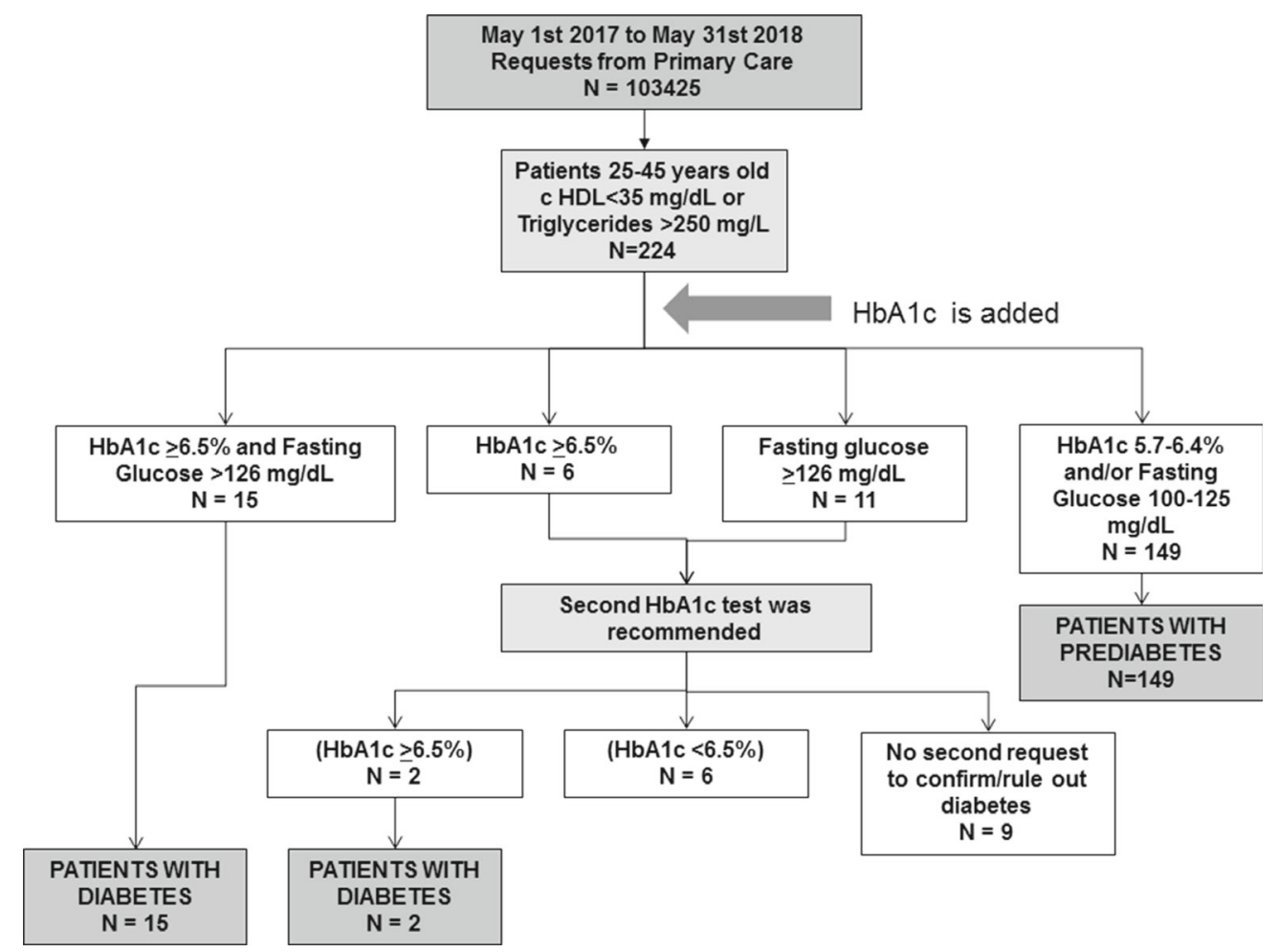

Fig. 1 Strategy to detect new cases of type 2 diabetes and prediabetes conditions. High-density lipoprotein cholesterol $\quad(\mathrm{cHDL})<35 \mathrm{mg} / \mathrm{dl} \quad(0.90 \mathrm{mmol} / \mathrm{l})$. Triglycerides $>250 \mathrm{mg} / \mathrm{dl}(2.83 \mathrm{mmol} / \mathrm{l})$. Glycated hemoglobin

population and can help to monitor the disease in compliance with guidelines $[1,2,16]$.

Diabetes is reaching epidemic proportions worldwide. Any efforts to ensure early diagnosis and correct management will have a major impact on the long-term health of the diabetic population and consequently on health costs. There are no "magic bullets," but the multiple interventions of all stakeholders involved in the diagnosis and management of this disease could overall result in major improvements in care [17].

The early identification of individuals with diabetes is a crucial action to reduce the overall burden of the disease [18]. Early detection and therefore prompt initiation of treatment can delay the progression of the disease and reduce the risk of complications related to diabetes [19]. We previously presented an intervention to identify subjects through a prospective measurement of HbA1c in every primary care patient $>45$ years old, at a cost of $85.4 €$ per
(HbAlc): $\geq 6.5 \%(48 \mathrm{mmol} / \mathrm{mol}) ;$ between $5.7 \%$ and $6.4 \%$ (39-46 mmol $/ \mathrm{mol})$; fasting glucose: $126 \mathrm{mg} / \mathrm{dl}$ (7 mmol/l); between $100 \mathrm{mg} / \mathrm{dl}$ and $125 \mathrm{mg} / \mathrm{dl}$ $(5.6-6.9 \mathrm{mmol} / \mathrm{l})$

identified case [13]. Although we did not take into the account the potential additional costs of earlier diagnosis, in terms of health care provision (medication, increased appointments, etc.), the current intervention is even more efficient because the cost of detected cases is lower and younger individuals are identified, making this strategy very attractive.

Once again, through investments in the clinical laboratory via the automated registration of additional laboratory tests, we are improving patient diagnosis [13, 20, 21] and treatment [21].

The diabetes guidelines are used to prevent diabetes-related morbidity by preventing complications such as retinopathy, nephropathy and neuropathy. However, guidelines are not correctly followed [9]. Numerous studies on cardiovascular risk reduction have demonstrated that better glycemic and lipid control can substantially reduce the risk of complications from diabetes [22-24]. Additionally, there 
Table 2 Requests with diabetes diagnosis adjusted according to clinical guidelines

\begin{tabular}{lll}
\hline & $\begin{array}{l}\text { Number of } \\
\text { tests } \\
\text { automatically } \\
\text { registered }\end{array}$ & $\begin{array}{l}\text { Median (P25-P75) } \\
\text { of results }\end{array}$ \\
\hline HbA1c & 91 & $6.8 \%(6.2 \%-7.8 \%)$ \\
Lipid profile & 708 & $51(44-62) \mathrm{mmol} / \mathrm{mol}$ \\
& Cholesterol & $172(149-195) \mathrm{mg} / \mathrm{dl}$ \\
& & $4.5(3.9-5.1) \mathrm{mmol} / \mathrm{l}$ \\
& cHLD & $47.5(39.2-58.5) \mathrm{mg} / \mathrm{dl}$ \\
& & $1.2(1.0-1.5) \mathrm{mmol} / \mathrm{l}$ \\
& cLDL & $94.0(76.0-115.0) \mathrm{mg} / \mathrm{dl}$ \\
& & $2.4(2.0-3.0) \mathrm{mmol} / \mathrm{l}$ \\
& Triglycerides & $121.0(89.0-168.0) \mathrm{mg} / \mathrm{dl}$ \\
& & $1.4(1.0-1.9) \mathrm{mmol} / \mathrm{l}$ \\
& 862 & $15.5(6.7-48.2) \mathrm{mg} / \mathrm{g} \mathrm{Crea}$ \\
& & $1.8(0.8-5.5) \mathrm{mg} / \mathrm{mmol}$ \\
& & Crea \\
\hline
\end{tabular}

$A C R$ albumin-to-creatinine ratio

is a proven relationship between healthy lifestyle behaviors and cardiovascular risk factors [25]. An early assessment and control of diabetes and adequate control of lipid levels may delay the progression of vascular complications and consequently improve patient outcomes [22-24, 26, 27].

However, in our cohort, similar to those reported in other studies in Spain [28] and in other countries [29], HbA1c and lipid tests, and especially ACR analyses, are under-requested, which is totally inappropriate for controlling and preventing kidney complications [30]. It is difficult to know why primary care physicians, who are at the front line of diabetes care, do not follow guidelines on a daily basis. Factors such as limited consultation time due to an overwhelming burden of patients or difficulties in the management of information systems could constitute major impediments to meeting diabetes guidelines.
As we could not describe type 2 diabetes detected by GPs in the same study period, we do not know how closely the guidelines have been followed. However, according our study results, it is far from $100 \%$.

Through our strategies, we achieved a request that complied with the guidelines, which could improve the course of the disease. However, the results of our study could have even more clinical implications since it is known that patients with high comorbidity rates are are less monitored in terms of HbA1c and cLDL levels [7]. Considering this, we could improve monitoring of the diabetes population with high comorbidity rates.

The study had some limitations. Regarding the first objective, we could have missed diagnoses of diabetes as we never received the second sample needed to confirm/discard diagnoses of the disease in nine cases. Moreover, we could not describe type 2 diabetes detected by GPs in the same study period. Regarding the second objective, we also could have missed data because including the reason for testing is optional for GPs. Another limitation was that only process metrics were evaluated in the study; we could not study whether the improvement in diabetes detection and monitoring positively affected patient outcome. Moreover, replication of the study would depend of the health care system organization, and therefore transferability of the results observed here to other countries than Spain could be difficult. Finally, our project could have taken a broader view of type 2 diabetes treatment, and too much information is still lacking for the calculated economic savings, as we only included reagent costs, but not time and other variables, and this may not apply to other countries or settings, since our laboratory belongs to the Public Health Network, where reagent prices are very low.

\section{CONCLUSION}

In conclusion, the laboratory can play a crucial role in the diagnosis and management of diabetes by using laboratory technology and designing and implementing strategies for a better adherence to guidelines. 


\section{ACKNOWLEDGEMENTS}

We thank the participants of the study.

Funding. No funding or sponsorship was received for this study or publication of this article. The article processing charges were funded by the authors.

Authorship. All named authors meet the International Committee of Medical Journal Editors (ICMJE) criteria for authorship for this article, take responsibility for the integrity of the work as a whole, and have given their approval for this version to be published.

List of Investigators. Members of the PRIMLAB working group are the following: Beatriz Massa-Dominguez (Chief Executive); Antonio Gómez-Gras (Primary Care Director); Norma Iranzo (Medical vice-director); Alejandro Canals-Baeza (CS Santa Faz-Ayuntamiento); Jose L López-Blasco (CS Hospital Provincial-Plá); Esperanza Gonzalez-Alvaro (CS Juan XXIII); Ramon de Juan Francés (CS Cabo Huertas); Jordi Gisbert-Segura (CS Campello); Rosario OliverRos (CS Xixona); Alberto Asencio-Aznar (CS Mutxamel); Juan J Ballester-Baixaulí (CS Sant Joan) and Carmen Pardo-Tomas (CSI Gerona).

Disclosures. Maria Salinas, Maite LópezGarrigós, Emilio Flores, Javier Lugo and Carlos Leiva-Salinas have no disclosures.

Compliance with Ethics Guideline. All procedures performed in studies involving human participants were in accordance with Hospital San Juan Research Committee and with the 1964 Helsinki Declaration and its later amendments or comparable ethical standards. The study was approved by the Hospital Research Committee, and the need for consent was waived.

Data Availability. The data sets during and/ or analyzed during the current study are available from the corresponding author on reasonable request.
Open Access. This article is distributed under the terms of the Creative Commons Attribution-NonCommercial 4.0 International License (http://creativecommons.org/licenses/ by-nc/4.0/), which permits any noncommercial use, distribution, and reproduction in any medium, provided you give appropriate credit to the original author(s) and the source, provide a link to the Creative Commons license, and indicate if changes were made.

\section{REFERENCES}

1. American Diabetes Association. Classification and diagnosis of diabetes: standards of medical care in diabetes-2018. Diabetes Care. 2018;41:S13-S27. https://doi.org/10.2337/dc18-S002.

2. Ministerio de Sanidad, Consumo y Bienestar Social-Ciudadanos-La Diabetes. 2018. https:// www.mscbs.gob.es/ciudadanos/enfLesiones/enfNo Transmisibles/diabetes/diabetes.htm. Accessed 14 Dec 2018

3. American Diabetes Association AD. Comprehensive medical evaluation and assessment of comorbidities: standards of medical care in diabetes-2018. Diabetes Care. 2018;41:S28-S37. https://doi.org/10. 2337/dc18-S003.

4. Salinas M, López-Garrigós M, Uris J, Leiva-Salinas C. A study of the differences in the request of glycated hemoglobin in primary care in Spain: a global, significant, and potentially dangerous under-request. Clin Biochem. 2014;47:1104-7. https://doi.org/10. 1016/j.clinbiochem.2014.04.020.

5. Salinas M, López-Garrigós M, Carratala A, et al. An evaluation of glycosylated hemoglobin requesting patterns in a primary care setting: a pilot experience in the Valencian Community (Spain). Endocrinol y Nutr órgano la Soc Española Endocrinol y Nutr. 2011;58:219-23. https://doi.org/10.1016/j.endonu. 2011.02.009.

6. Salinas M, Lopez-Garrigos M, Flores E, et al. Urinary albumin: a risk marker under-requested in primary care in Spain. Ann Clin Biochem. 2017. https://doi. org/10.1177/0004563217716475.

7. Buja A, Gini R, Visca M, et al. Need and disparities in primary care management of patients with diabetes. BMC Endocr Disord. 2014;14:56. https://doi. org/10.1186/1472-6823-14-56.

8. National Diabetes Audit Report 1-findings and recommendations 2016-17-NHS Digital. 2018. 
https://digital.nhs.uk/data-and-information/publicat ions/statistical/national-diabetes-audit/national-diab etes-audit-report-1-findings-and-recommendations2016-17. Accessed 3 Jul 2018

9. Hashmi NR, Khan SA. Adherence to diabetes mellitus treatment guidelines from theory to practice: the missing link. J Ayub Med Coll Abbottabad. 2016;28:802-8.

10. Ahola AJ, Forsblom C, Groop PH. Adherence to special diets and its association with meeting the nutrient recommendations in individuals with type 1 diabetes. Acta Diabetol. 2018;55:843-51. https:// doi.org/10.1007/s00592-018-1159-2.

11. Lugtenberg M, Burgers JS, Besters CF, et al. Perceived barriers to guideline adherence: a survey among general practitioners. BMC Fam Pract. 2011;12:98. https://doi.org/10.1186/1471-2296-12-98.

12. Salinas M, López-Garrigós M, Flores E, et al. Indications for laboratory tests in primary care: assessment of the most frequent indications and requests with blank clinical information. Biochem Med. 2016;26:431-5. https://doi.org/10.11613/BM.2016.045.

13. Salinas M, Lopez-Garrigos M, Flores E, et al. Automatic laboratory-based strategy to improve the diagnosis of type 2 diabetes in primary care. Biochem Med. 2016;26:121-8. https://doi.org/10. 11613/BM.2016.013.

14. Centers for disease control and prevention, National Center for Health statistics, 2013. ICDICD-9-CM-International Classification of Diseases, Ninth Revision. Classif Dis Funct Disabil: Clinical Modification; 2013.

15. Salinas M, López-Garrigós M, Gutiérrez M, et al. Two minutes of monthly monitoring can ensure quality laboratory service every day of the year. Lab Med. 2010;41:360-3. https://doi.org/10.1309/ LM8OI14LCORJYHVY.

16. Fisterra.com (2018) Diabetes mellitus tipo 2. www. fisterra.com/guias-clinicas/diabetes-mellitus-tipo-2/ \#26612. Accessed Feb 2019.

17. Grimshaw JM, Shirran L, Thomas R, et al (2001) Changing provider behavior: an overview of systematic reviews of interventions. Med Care 39:II2-45

18. Laiteerapong N, Cifu AS. Screening for prediabetes and type 2 diabetes mellitus. JAMA. 2016;315:697. https://doi.org/10.1001/jama.2015.17545.

19. Marchesini G, Pasqualetti P, Anichini R, et al. Patient preferences for treatment in type 2 diabetes: the Italian discrete-choice experiment analysis. Acta Diabetol. 2018. https://doi.org/10.1007/ s00592-018-1236-6.
20. Salinas M, López-Garrigós M, Pomares F, et al. Serum calcium ( $\mathrm{S}-\mathrm{Ca}$ ), the forgotten test: preliminary results of an appropriateness strategy to detect primary hyperparathyroidism (pHPT). Bone. 2013;56:73-6.

21. Salinas M, Flores E, López-Garrigós M, et al. Computer-assisted interventions in the clinical laboratory process improve the diagnosis and treatment of severe vitamin $\mathrm{B}_{12}$ deficiency. Clin Chem Lab Med. 2018. https://doi.org/10.1515/cclm-2017-1116.

22. Nathan DM, Genuth S, Diabetes Control and Complications Trial Research Group et al (1993) The effect of intensive treatment of diabetes on the development and progression of long-term complications in insulin-dependent diabetes mellitus. N Engl J Med 329:977-986. https://doi.org/10.1056/ NEJM199309303291401

23. UK Prospective Diabetes Study (UKPDS) Group. Intensive blood-glucose control with sulphonylureas or insulin compared with conventional treatment and risk of complications in patients with type 2 diabetes (UKPDS 33). Lancet. 1998;352:837-53.

24. Grundy SM, Cleeman JI, Bairey Merz CN, et al. Implications of recent clinical trials for the National Cholesterol Education Program Adult Treatment Panel III Guidelines. J Am Coll Cardiol. 2004;44:720-32. https://doi.org/10.1016/j.jacc.2004.07.001.

25. Huang Y, Li J, Zhu X, et al. Relationship between healthy lifestyle behaviors and cardiovascular risk factors in Chinese patients with type 2 diabetes mellitus: a subanalysis of the CCMR-3B study. Acta Diabetol. 2017;54:569-79. https://doi.org/10.1007/ s00592-017-0981-2.

26. Gavin JR, Freeman JS, Shubrook JH, Lavernia F (2011) Type 2 diabetes mellitus: practical approaches for primary care physicians. J Am Osteopath Assoc 111:S3-12 (quiz S13)

27. American Diabetes Association. Standards of medical care in diabetes-2018 abridged for primary care providers. Clin Diabetes. 2018;36:14-37. https:// doi.org/10.2337/cd17-0119.

28. Menéndez E, Delgado E, Fernández-Vega F, et al. Prevalencia, diagnóstico, tratamiento y control de la hipertensión arterial en España. Resultados del estudio Di@bet.es. Rev Española Cardiol. 2016;69:572-8. https://doi.org/10.1016/j.recesp.2015.11.036.

29. WHO. Global report on diabetes. Geneva: WHO; 2017.

30. CKD Work Group. KDIGO 2012 Clinical practice guideline for the evaluation and management of chronic kidney disease. Kidney Int. 2013;3:4-4. https://doi.org/10.1038/kisup.2012.76. 